\title{
Perfil epidemiológico da tuberculose no Estado do Piauí no período de 2015 a 2020
}

\author{
Epidemiological profile of tuberculosis in the State of Piauí in the period from 2015 to 2020 \\ Perfil epidemiológico de tuberculosis en el Estado de Piauí en el período de 2015 a 2020
}

Recebido: 12/07/2021 | Revisado: 19/07/2021 | Aceito: 20/07/2021 | Publicado: 03/08/2021

\author{
Gabriel Ferreira de Sousa \\ ORCID: https://orcid.org/0000-0003-4323-4408 \\ Centro Universitário UniFacid, Brasil \\ E-mail: gabriel.biomed94@gmail.com \\ Alice Lima Rosa Mendes \\ ORCID: https://orcid.org/0000-0002-1960-9647 \\ Universidade de Brasília, Brasil \\ E-mail: alice_lima_@hotmail.com \\ Gabriela Dantas Carvalho \\ ORCID: https://orcid.org/0000-0002-9571-3323 \\ Centro Universitário UniFacid, Brasil \\ E-mail: ftgabrieladantas@hotmail.com \\ Suely Moura Melo \\ ORCID: https://orcid.org/0000-0001-9996-0850 \\ Centro Universitário Unifacid, Brasil \\ E-mail: suelymouramelo@yahoo.com.br \\ Rayssa Maria de Araujo Carvalho \\ ORCID: https://orcid.org/0000-0002-2567-0209 \\ Centro Universitário UniFacid, Brasil \\ E-mail: rayssacarv@gmail.com
}

\begin{abstract}
Resumo
A tuberculose é uma doença infectocontagiosa de grande importância da saúde pública, causada pelo Mycobacterium tuberculosis, sendo transmitida através de aerossóis expelidos no ambiente pela fala, tosse ou espirro, advindos de um indivíduo previamente infectado. $\mathrm{O}$ objetivo deste estudo foi definir um perfil epidemiológico da tuberculose no estado do Piauí, durante os anos de 2015 a 2020. Tratou-se de um estudo epidemiológico descritivo de série histórica, quantitativo e documental, apresentando uma análise de saúde. Os dados foram obtidos no Sistema de Informação de Agravos de Notificação (SINAN), acerca dos casos notificados de tuberculose de pessoas residentes no estado do Piauí. Houve registros de $416(9,01 \%)$ casos de óbitos de pessoas acometidas por tuberculose, no período de 2015 a 2020 , sendo entre estes $168(3,63 \%)$ causados pela tuberculose e $251(5,43 \%)$ por outras causas. De acordo com os dados obtidos no sistema sobre o período estudado, confirma-se que o estado do Piauí é uma área com alta carga de tuberculose, de transmissão acentuada e que a doença está associada às condições socioeconômicas dos indivíduos, necessitando de um melhor planejamento pelos órgãos de saúde do estado. O trabalho se propõe a auxiliar o Plano Nacional de Controle a Tuberculose e pode servir de base para ajudar na melhoria das estratégias para combater e controlar com mais eficácia a doença na região do Piauí.
\end{abstract}

Palavras-chave: Tuberculose; Perfil epidemiológico; Mycobacterium tuberculosis.

\begin{abstract}
Tuberculosis is an infectious disease of great public health importance, caused by Mycobacterium tuberculosis, being transmitted through aerosols expelled into the environment through speech, coughing or sneezing, coming from a previously infected individual. The objective of this study was to define an epidemiological profile of tuberculosis in the state of Piauí, from 2015 to 2020. It was a descriptive epidemiological study of historical, quantitative and documentary series, presenting an analysis of health. Data were obtained from the Notifiable Diseases Information System (SINAN), about notified cases of tuberculosis in people residing in the state of Piauí. There were records of $416(9.01 \%)$ cases of death of people affected by tuberculosis, in the period 2015 to 2020, among these 168 (3.63\%) were caused by tuberculosis and $251(5.43 \%)$ by other causes. According to the data obtained in the system over the period studied, it is confirmed that the state of Piauí is an area with a high burden of tuberculosis, of accentuated transmission and that the disease is associated with the socioeconomic conditions of individuals, requiring better planning by the state health agencies. The work is intended to assist the National Plan for Tuberculosis Control and can serve as a basis to help improve strategies to combat and control the disease more effectively in the region of Piauí.
\end{abstract}

Keywords: Tuberculosis; Epidemiological profile; Mycobacterium tuberculosis. 


\section{Resumen}

La tuberculosis es una enfermedad infecciosa de gran importancia para la salud pública, causada por Mycobacterium tuberculosis, que se transmite a través de aerosoles expulsados al medio ambiente a través del habla, tos o estornudos, provenientes de un individuo previamente infectado. El objetivo de este estudio fue definir un perfil epidemiológico de la tuberculosis en el estado de Piauí, de 2015 a 2020. Fue un estudio epidemiológico descriptivo de series históricas, cuantitativas y documentales, presentando un análisis de salud. Los datos se obtuvieron del Sistema de Información de Enfermedades Notificables (SINAN), sobre casos notificados de tuberculosis en personas residentes en el estado de Piauí. Se registraron $416(9,01 \%)$ casos de defunción de personas afectadas por tuberculosis, en el período 2015 a 2020, de estos 168 (3,63\%) fueron causados por tuberculosis y $251(5,43 \%)$ por otras causas. De acuerdo con los datos obtenidos en el sistema durante el período estudiado, se confirma que el estado de Piauí es una zona con alta carga de tuberculosis, de transmisión acentuada y que la enfermedad está asociada a las condiciones socioeconómicas de los individuos, requiriendo una mejor planificación. Por las agencias estatales de salud. El trabajo está destinado a ayudar al Plan Nacional de Control de la Tuberculosis y puede servir como base para ayudar a mejorar las estrategias para combatir y controlar la enfermedad de manera más efectiva en la región de Piauí.

Palabras clave: Tuberculosis; Perfil epidemiológico; Tuberculosis micobacteriana.

\section{Introdução}

A tuberculose (TB) é uma doença de grande importância na saúde pública, que comumente é causada pela espécie Mycobacterium tuberculosis que também é conhecido como Bacilo de Koch (BK), porém, também pode ser ocasionada por outras espécies do mesmo gênero, como a M. bovis, M. africanum, M. canetti, M. microti, M. pinnipedi e M. caprae. Esta doença é infectocontagiosa, sendo propagada pelas vias aéreas, advindo de alguma pessoa previamente infectada com algum dos tipos de Mycobacterium e que, através da tosse, espirro ou a fala, irão exalar no ambiente aerossóis que contenham bacilos (Brasil, 2019); (Frans, 2013).

A TB ainda está na lista das 10 principais causas de morte no mundo, registrando cerca de 10 milhões de novos casos por ano e pouco mais de 1 milhão de mortos, destes, pouco mais de 73 mil novos casos foram registrados no Brasil só em 2019. Ainda que tenha cura para a TB, a desistência do tratamento se torna a causa principal de vítimas fatais, tendo em conta que em 2018 foram registrados 4.490 óbitos por TB somente no Brasil (Frasão, 2020).

Apesar dos números citados, o Brasil ainda é referência mundial no controle frente à TB, onde, na lista dos 30 países com alta carga dos agentes infecciosos, o país apresentou os menores números de incidência e mortalidade através do desenvolvimento das ações de combate à TB, como programas de prevenção, ampliação de cobertura da vacina BCG além de recomendações de investigação frente aos casos latentes de $M$. tuberculosis, onde os investimentos estruturais e tecnológicos no Sistema Único de Saúde (SUS) foram determinantes e contribuíram para a redução da morbidade (Barreira, 2018); (Brasil 2020); (Who, 2017).

Dentro desse contexto, esta pesquisa tem como objetivo geral avaliar o perfil epidemiológico de portadores de Tuberculose no Piauí, entre 2015 e 2020 bem como os objetivos específicos identificar o sexo, a raça, a faixa etária, escolaridade, zona residência, forma, coinfecção TB/HIV e situação encerrada dos casos de TB no Piauí.

A relevância desse estudo, serve para destacar as medidas tomadas nos últimos anos, onde o país se tornou referência no combate à $\mathrm{TB}$, e constatar se a vigilância aplicada à esta doença trouxe redução no número de casos, para assim verificar a eficácia do desenvolvimento das ações de combate à TB.

\section{Metodologia}

O presente estudo investigou o perfil epidemiológico dos casos de tuberculose que ocorreram no Piauí durante os anos de 2015 a 2020, obedecendo às questões éticas e legais preconizadas pela Resolução 466/2012 e 510/2016 do Conselho Nacional de Saúde. Como não foi abordada uma assistência aos pacientes e utilizou-se, neste estudo, apenas dados coletados diretamente do Sistema de Informação de Agravo de Notificação (SINAN), em que não há a identificação do paciente, e os dados são de domínio público, o mesmo não foi submetido ao Comitê de Ética em Pesquisa (CEP). Esta pesquisa trata-se de 
um estudo retrospectivo, epidemiológico, documental, de abordagem quantitativa e caráter exploratório e descritivo.

O presente estudo foi realizado envolvendo todos os casos de tuberculose ocorridos no estado do Piauí e registrados no DATASUS - (SINAN), no período de 2015 a 2020, sendo possível analisar como critérios de inclusão e os critérios de exclusão abrangeram todos os casos de pacientes que tiveram tuberculose diagnosticada e registrados pelo (SINAN), mas que não eram moradores do estado do Piauí. No banco de dados, constam as informações obtidas através da ficha de notificação compulsória do Sistema de Informação de Agravos de Notificação (SINAN) - (Anexo A).

O objetivo do SINAN é coletar, transmitir e disseminar dados gerados rotineiramente pelo sistema de vigilância epidemiológica das três esferas de governo, por intermédio de uma rede informatizada para apoiar o processo de investigação e dar subsídios à análise das informações de vigilância epidemiológica das doenças de notificação compulsória.

Os dados foram coletados do DATASUS - (SINAN), através de tabelas e gráficos fornecidos pelo sistema, e então transpostos para planilhas do programa Microsoft Excel ${ }^{\circledR}$ 2016, durante o mês de junho de 2021. Foi realizada uma análise de todos os casos de tuberculose do estado no Piauí entre 2015 e 2020, onde buscou-se identificar as variáveis como sexo, raça, faixa etária, escolaridade, zona residência, forma, coinfecção TB/HIV e situação encerrada.

A análise foi realizada através de estatística descritiva simples, com base em números absolutos e porcentagens. Os resultados foram mostrados através de tabelas e gráficos, para melhor compreensão.

\section{Resultados e Discussão}

Segundo o SINAN, entre os anos de 2015 e 2020, foram notificados um total de 4.616 casos de TB no estado do Piauí, onde o menor número de casos foi no ano de 2015 com 704 (15,25\%) casos e o maior foi no ano de 2018 com 841 $(18,22 \%)$ casos (Gráfico 1).

Gráfico 1 - Número de casos confirmados de tuberculose segundo o ano de ocorrência no Piauí de 2015 a 2020.

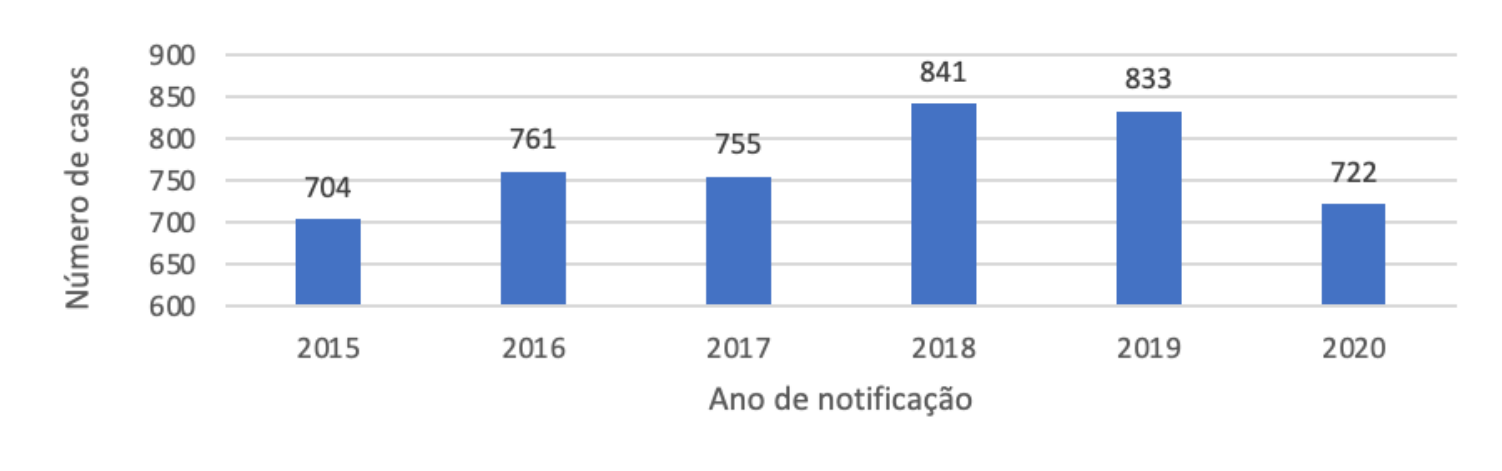

Fonte: Ministério da Saúde/SVS - Sistema de Informação de Agravos de Notificação - (SINAN) Net.

O número de casos entre os anos de 2015 e 2017 tiveram pouca variação, entretanto, nos anos de 2018 e 2019 houve um aumento expressivo no número de casos e em seguida uma diminuição em 2020, voltando à faixa de variação que houve entre 2015 e 2017. A média de casos no período em pesquisa foi de 769 casos por ano, o que evidencia um elevado número.

Um estudo feito entre os anos de 2010 e 2015 no mesmo estado, apresentou 4.914 casos, onde os números de casos confirmados possuem uma certa semelhança com o estudo em vigência, constatando que a tuberculose no estado do Piauí vem há anos com uma variável constante de casos confirmados (Barreto, 2020).

Analisando o número de casos da TB segundo o sexo, observa-se uma predominância da doença no sexo masculino com 2.960 casos confirmados, com um percentual representativo de 64,12\%, e o sexo feminino contando com os outros 1.656 casos, correspondendo apenas a 35,88. Ressalta-se o aumento de casos no sexo masculino ao longo dos anos de 2015 a 2019 , 
onde em 2015 foram registrados 434 casos e em 2019, 558 casos, como pode ser verificado no Gráfico 2.

Gráfico 2 - Número de casos confirmados de tuberculose, segundo o sexo e ano de ocorrência no Piauí de 2015 a 2020.

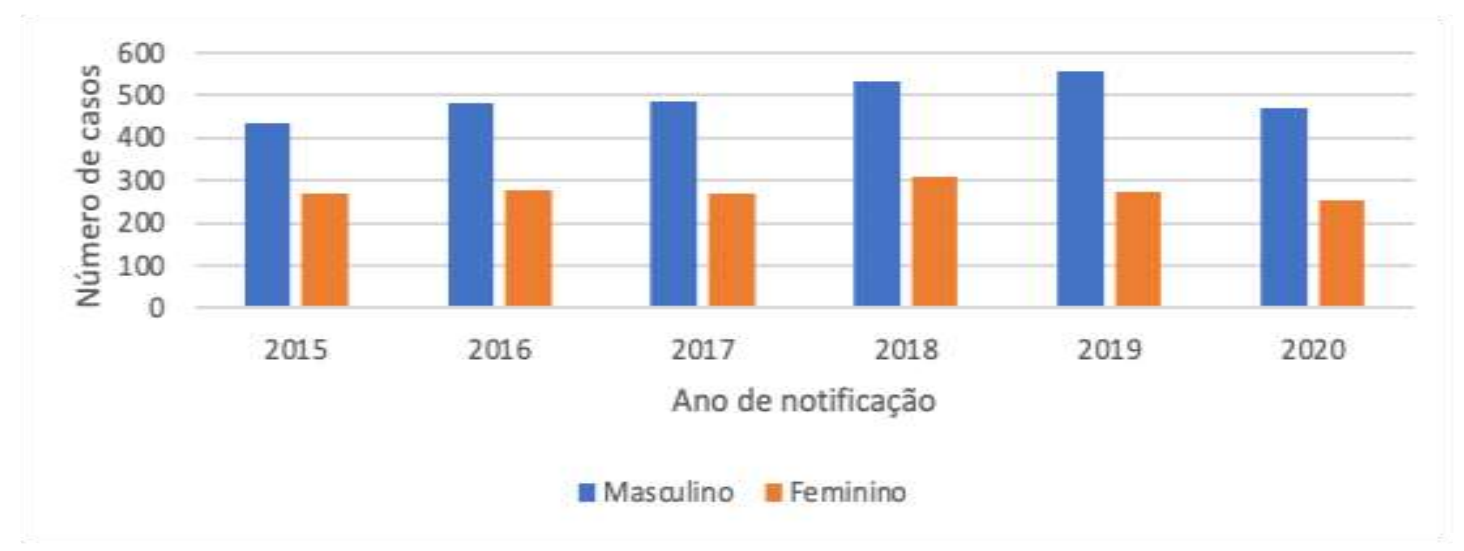

Fonte: Ministério da Saúde/SVS - Sistema de Informação de Agravos de Notificação - (SINAN) Net.

Um estudo realizado em 2018 no estado de Goiás, registrou 1.200 casos de tuberculose, sendo que destes, 888 (74\%) casos foram em indivíduos do sexo masculino e 312 (26\%) no sexo feminino, o que fica evidente a alta prevalência relacionada ao sexo masculino, bem como na pesquisa em vigência (Silva, 2018).

Segundo (Nobre et al., 2019), realizaram uma pesquisa dos casos de tuberculose no estado do Ceará entre 2014 e 2018, onde foram registrados só na cidade de Fortaleza, 9.742 casos, destes, 6.260 (64,25\%) casos foram registrados em indivíduos do sexo masculino e 3.482 (35,74\%) casos no sexo feminino, ressaltando a predominância em indivíduos do sexo masculino e se equiparando com os resultados obtidos. Entretanto não existe um motivo específico relacionando a alta prevalência de casos em indivíduos do sexo masculino, apenas sugere-se que, uma maior exposição a agentes infecciosos, situações de risco como bebidas alcoólicas e fumos, bem como a menor procura destes indivíduos pelos serviços de saúde, podem refletir nestes resultados (Zagmignan et al., 2014).

Quando analisado o grau de escolaridade dos pacientes com tuberculose durante o período estudado, observa-se que a falta de medidas ou instruções aos pacientes pode estar relacionada com a infecção, pois o intervalo da $1^{\mathrm{a}}$ a $4^{\mathrm{a}}$ série incompleta se apresenta com 891 (19,30\%) casos e que as notificações em que não se aplicam o registro de escolaridade dos pacientes, representou o menor número, sendo notificado somente 49 (1,06\%), seguido dos pacientes com ensino superior incompleto, que teve 84 (1,82\%) casos (Gráfico 3). Apesar do elevado número, o intervalo da $1^{\mathrm{a}}$ a $4^{\mathrm{a}}$ apresentou uma redução no número dos casos ao longo do período de estudo, registrando 161 casos em 2016 e 129 em 2020. 
Gráfico 3 - Número de casos confirmados de tuberculose, segundo a escolaridade e ano de ocorrência no Piauí de 2015 a 2020 .

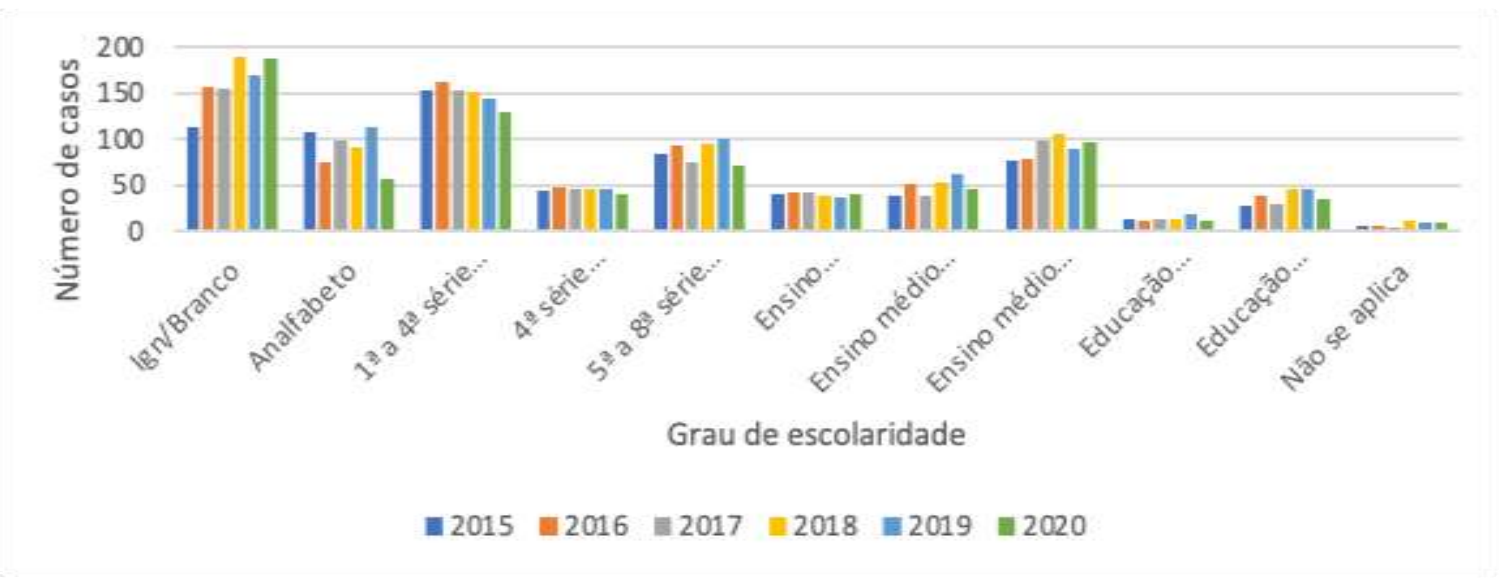

Fonte: Ministério da Saúde/SVS - Sistema de Informação de Agravos de Notificação - (SINAN) Net.

$\mathrm{O}$ acometimento às doenças transmissíveis, como a tuberculose, é mais prevalente em indivíduos com baixa atividade econômica, uma vez que estes têm menos acesso à educação em saúde. Um estudo feito com os casos registrados de tuberculose no Brasil ente 2012 e 2016, mostrou que a tuberculose não só está relacionada ao sexo masculino, como também à baixa escolaridade ou nenhuma, onde foram notificados 231.186 (17,29\%) casos em indivíduos analfabetos, bem como $189.230(14,15 \%)$ casos em indivíduos no intervalo da $5^{\circ}$ a $8^{\circ}$ série incompleta do ensino fundamental (Fontes et al.,2019).

Em relação às raças, nota-se o maior número de casos presente na raça parda com 3.274 casos diagnosticados (70,93\%), e o menor número na raça indígena, registrando apenas $8(0.17 \%)$ casos durante o período da pesquisa (Gráfico 4).

Gráfico 4 - Número de casos confirmados de tuberculose, segundo a raça e ano de ocorrência no Piauí de 2015 a 2020.

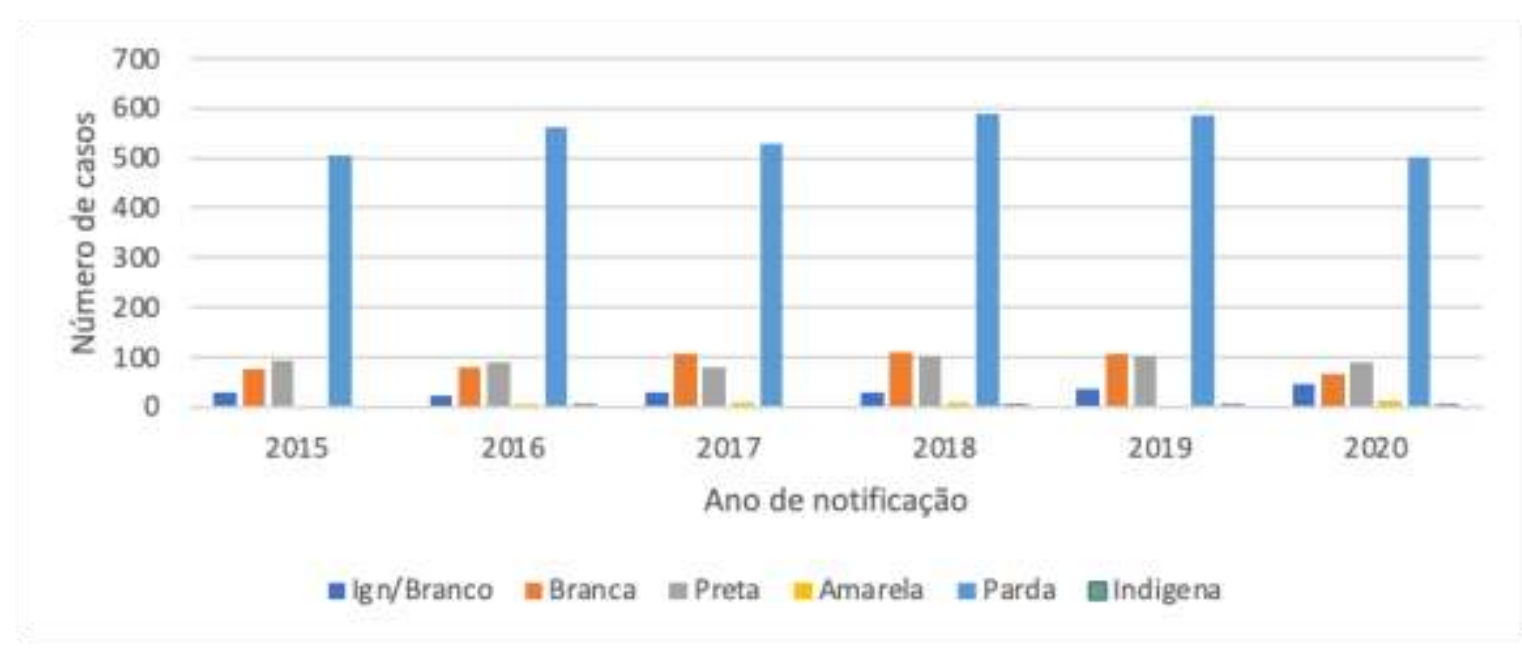

Fonte: Ministério da Saúde/SVS - Sistema de Informação de Agravos de Notificação - (SINAN) Net.

Entre 2008 e 2018, um estudo registrou 4.201 casos no DF, e destes, foram registrados 63\% dos casos à população negra e 52\% à população parda, evidenciando que a tuberculose independe de raça/etnia (Frasão, 2020).

Outro estudo, no período de 2012 a 2016 no estado do Maranhão, registrou 9.492 casos, sendo destes, 6.698 (70,5\%) casos foram relacionados à população parda, assemelhando-se com o estudo vigente (70,93\%), (Oliveira, 2018).

A alta prevalência dos casos de tuberculose associada a indivíduos autodeclarados pardos, pode estar relacionada com a situação econômica destes indivíduos, onde fatores como míseros e residir em zonas urbanas, facilitam a disseminação do 
BK e também uma piora do quadro clínico dos indivíduos já acometidos. A mistura de raças instalada no Brasil também pode influenciar na decisão dos indivíduos se autodeclararem pardos, resultando em um maior número de casos relacionado a essa etnia (Dantas et al., 2019).

Os números de casos são mais frequentes na faixa etária de 20 a 39 anos com 1.635 (35,42\%) casos e na faixa de 40 a 59 anos, apresentando 1.552 (33,64\%) casos diagnosticados. Durante o período de estudo, pode-se evidenciar também uma ascendência no número de casos ano após ano ente 2015 e 2019 na faixa etária de 20 a 39, com uma redução de notificações apenas em 2020 e a faixa etária de 40 a 59 anos, apresentando os maiores números de casos por ano durante a pesquisa em 2018 e 2019 com 312 e 314 casos respectivamente (Gráfico 5).

Gráfico 5 - Número de casos confirmados de tuberculose, segundo a faixa etária e ano de ocorrência no Piauí de 2015 a 2020.

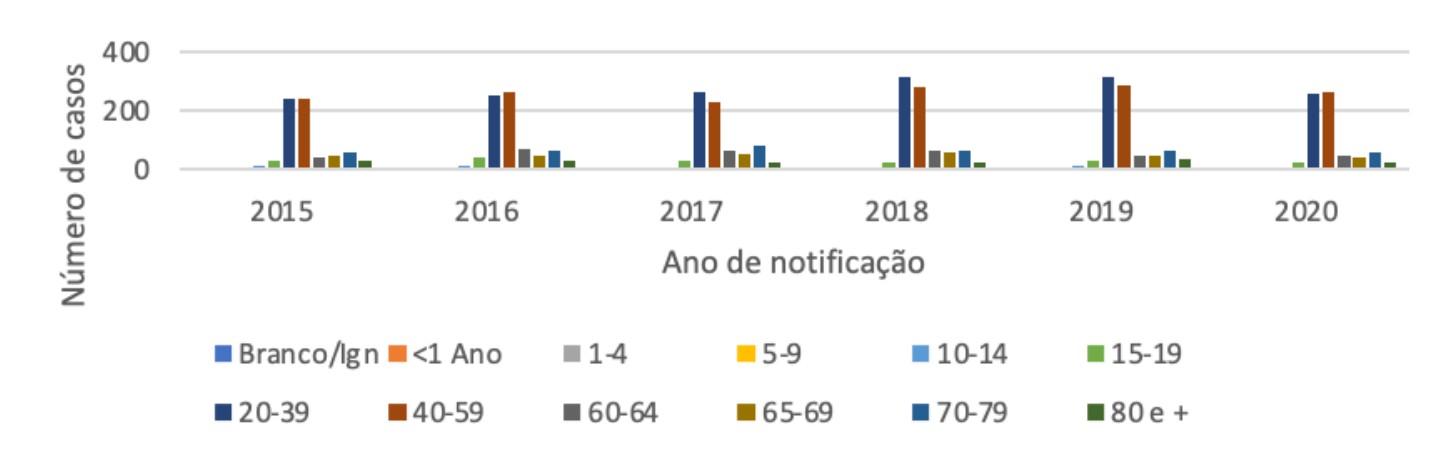

Fonte: Ministério da Saúde/SVS - Sistema de Informação de Agravos de Notificação - (SINAN) Net.

Alves et al,. 2017, avaliaram o número de casos no Piauí, no período de 2010 a 2014, onde houve o registro de 4.847 notificações, em que 1.665 (35\%) casos foram associados à faixa etária de 40 a 59 anos, e 1.624 (33,5\%) casos na faixa etária de 20 a 39 anos, o que mostra a consistência dos casos no estado relacionados às faixas etárias relatadas na pesquisa atual.

Ambas as faixas etárias são relacionadas com a fase adulta, onde as exposições por questões socioculturais ou trabalhistas além da necessidade de maior circulação em locais com um elevado número de pessoas da mesma faixa etária, podem resultar nos números apresentados, bem como também facilita a propagação dos bacilos nos ambientes frequentados pelos infectados (Barros et al. 2014); (Oliveira et al. 2021). Além disso, a diminuição do número de notificações em 2020 pode estar relacionada com a pandemia do coronavírus, onde a redução no consumo dos cartuchos de TRM para tuberculose, pode indicar a redução de diagnósticos realizados durante o ano (Vidal, 2021).

A relação da zona residência com os casos de tuberculose apresentou uma predominância de casos na zona urbana com $3.487(75,54 \%)$ casos, seguida da zona rural, com 935 (20,25\%) casos. Os ign/branco apresentaram $186(4,02 \%)$ casos e a zona periurbana registrou apenas $8(0,17 \%)$ casos como mostra a Tabela 1. 
Tabela 1 - Número de casos confirmados de tuberculose, segundo a zona residência e ano de ocorrência no Piauí de 2015 a 2020 .

\begin{tabular}{|c|c|c|c|c|c|c|}
\hline \multirow[t]{2}{*}{$\begin{array}{c}\text { Ano } \\
\text { de Notificação }\end{array}$} & \multirow[b]{2}{*}{ ranco } & Ign/B & $\mathbf{U}$ & \multirow{2}{*}{\multicolumn{2}{|c|}{ Periu }} & \multirow[b]{2}{*}{ otal } \\
\hline & & rbana & ural & & & \\
\hline \multirow[t]{2}{*}{2015} & 22 & & 5 & & 1 & \\
\hline & & 21 & 60 & & & 04 \\
\hline \multirow[t]{2}{*}{2016} & 27 & & 5 & & 1 & \\
\hline & & 90 & 43 & & & 61 \\
\hline \multirow[t]{2}{*}{2017} & 33 & & 5 & & 2 & \\
\hline & & 80 & 40 & & & 55 \\
\hline \multirow[t]{2}{*}{2018} & 24 & & 6 & & 1 & \\
\hline & & 33 & 83 & & & 41 \\
\hline \multirow[t]{2}{*}{2019} & 41 & & 6 & & 1 & \\
\hline & & 25 & 66 & & & 33 \\
\hline \multirow[t]{2}{*}{2020} & 39 & & 5 & & 2 & \\
\hline & & 38 & 43 & & & 22 \\
\hline \multirow[t]{2}{*}{ Total } & 186 & & 3 & $!$ & 8 & \\
\hline & & 487 & 35 & & & 616 \\
\hline
\end{tabular}

Legenda: Ign (Ignorado). Fonte: Ministério da Saúde/SVS - Sistema de Informação de Agravos de Notificação - (SINAN) Net.

Um estudo feito em Caxias no Maranhão, entre os anos de 2006 e 2016, registrou que 474 (83,2\%) casos foram diagnosticados em pacientes que residem na zona rural da cidade, e a zona rural apresentou apenas 96 (16,8\%) casos. O estudo evidencia ainda que o rápido processo de urbanização e outros fatores como poluição do ar, e concentração elevada de pessoas, podem contribuir para os maiores registros serem verificados nas zonas urbanas (Macedo et al., 2018).

Com relação às formas de tuberculose, os casos foram diagnosticados com maior frequência na forma pulmonar, registrando $3.809(82,51 \%)$ casos, já na forma extrapulmonar, foi registrado $688(14,90 \%)$ casos. O número de pacientes que apresentaram as duas manifestações clínicas de tuberculose, sendo ela pulmonar e extrapulmonar, foi de 188 (2.55\%) casos, e o ign/branco registrou apenas $1(0,02 \%)$ caso.

Gráfico 6 - Número de casos confirmados de tuberculose, segundo a forma e ano de ocorrência no Piauí de 2015 a 2020.

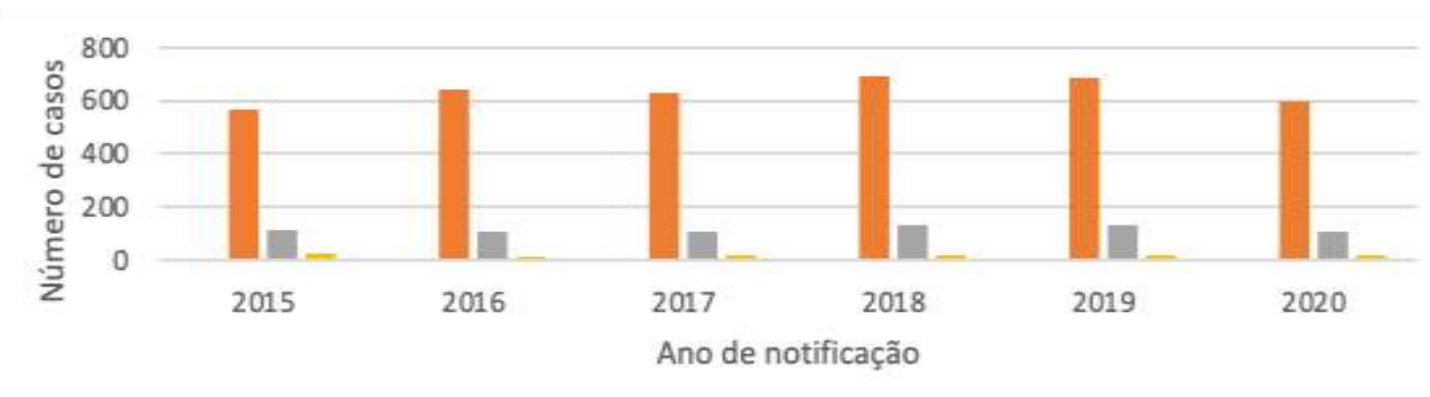

"ign/Branco $\equiv$ PULMONAR =EXTRAPULMONAR =PUMONAR + EXTRAPULMONAR

Fonte: Ministério da Saúde/SVS - Sistema de Informação de Agravos de Notificação - (SINAN) Net.

Vale ressaltar que além o BK infectar primeiramente os pulmões, devido às respostas monológicas, o bacilo pode se disseminar para outros órgãos, caracterizando a forma extrapulmonar da doença, onde, a detecção da forma clínica tem 
importância no diagnóstico, pois mudanças no tratamento podem ser feitas dependendo do órgão afetado pelo bacilo (Capone et al., 2006).

Uma pesquisa realizada no estado de Pernambuco no período de 2009 a 2019, que registrou 61.449 casos, onde 52.242 foram notificados na forma pulmonar da doença, totalizando $85,01 \%$, um valor semelhante ao relatado neste estudo (82.51\%), a forma extrapulmonar foi apresentada com 7.295 (11.87\%) casos e os casos de tuberculose pulmonar e extrapulmonar simultaneamente foram de 1.904 (3.09\%) casos notificados (Lima et al., 2021).

As análises de coinfecção TB/HIV mostram que do total de 4.616 casos presentes no período de estudo, 3.373 $(73,07 \%)$ sorologias para HIV foram solicitadas, onde $2.801(60,68 \%)$ dos casos apresentaram resultados negativos, 359 $(7,77 \%)$ casos resultaram em sorologia positiva, e 213 (4,61\%) casos foram registrados como "em andamento". Segundo os dados do (SINAN), 1.242 (26,90\%) casos não tiveram a sorologia para HIV realizada, e ign/branco registrou 1 (0,02\%) caso, como mostra o Gráfico 7.

Gráfico 7 - Número de casos confirmados de tuberculose, segundo a coinfecção TB/HIV e ano de ocorrência no Piauí de 2015 a 2020 .

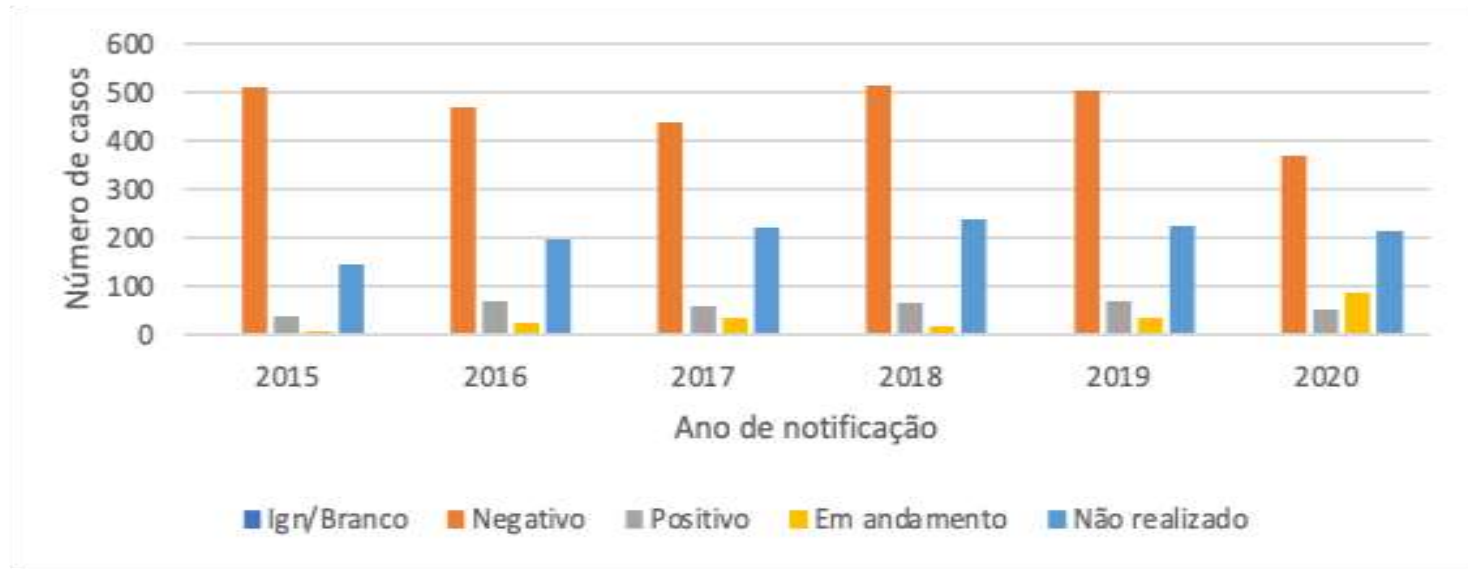

Fonte: Ministério da Saúde/SVS - Sistema de Informação de Agravos de Notificação - (SINAN) Net.

Entre 2009 e 2019, um estudo feto na cidade de Belém, foram notificados 20.054 casos no total, dentre estes, 2.596 $(12,94 \%)$ casos apresentaram sorologia positiva para HIV, resultando na confecção e evidenciando o alto número, em relação ao encontrado na pesquisa atual (Ferreira et al., 2021).

A infecção pelo HIV expõe o indivíduo a outras infecções, ente elas a do Mycobacterium tuberculosis, realizando a coinfecção TB/HIV, que pode aumentar as taxas de incidência e mortalidade, devido a imunodeficiência causada pelo HIV, além de que, a confecção TB/HV requer um tratamento adicional, que resultará em efeitos adversos e interações medicamentosas que demandam de um acompanhamento por conta da cronicidade da confecção (Magno et al., 2017); (De Miranda et al., 2017).

A evolução dos casos presentes no estudo de 2015 a 2020 mostra que $2.833(61,37 \%)$ casos foram curados, 168 $(3,63 \%)$ pacientes foram ao óbito por tuberculose e $251(5,43 \%)$ pacientes foram ao óbito por outras causas. Como mostra o Gráfico 8, $255(5,52 \%)$ pacientes abandonaram o tratamento e 28 (0,60\%) casos tiveram um abandono primário. Foram registrados $28(0,60 \%)$ casos como tuberculose droga resistente, e $27(0,58 \%)$ casos tiveram mudanças nos esquemas de tratamento, além de $5(0,10 \%)$ casos evoluírem para falência no tratamento. Os dados ainda mostram que 526 (11,39\%) casos foram transferidos e $525(11,37 \%)$ foram registrados como ign/brancos. 
Gráfico 8 - Número de casos confirmados de tuberculose, segundo a situação encerrada e ano de ocorrência no Piauí de 2015 a 2020.

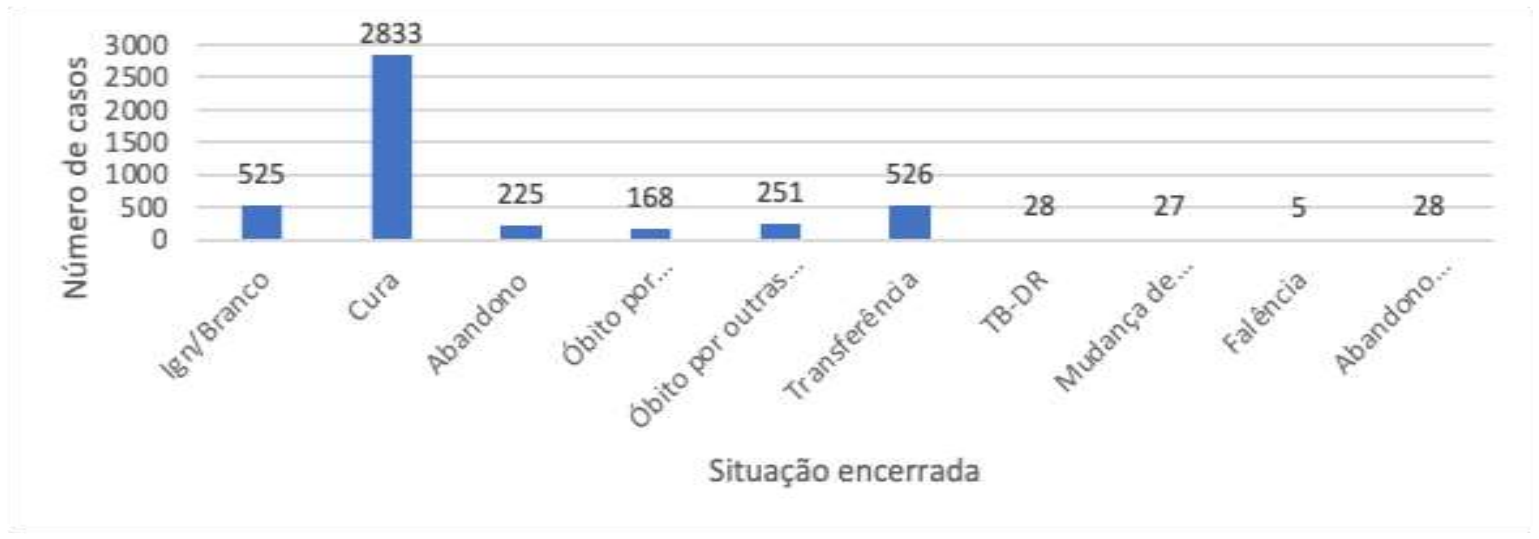

Fonte: Ministério da Saúde/SVS - Sistema de Informação de Agravos de Notificação - (SINAN) Net.

Na cidade de Sinop, Mato Grosso do Sul, no período de 2012 a 2018, uma pesquisa registrou 162 casos no total, onde destes $119(73,45 \%)$ casos evoluíram para cura e apenas 8 (4,9\%) foram registrados como abandonos no tratamento (Braga, 2019).

Nesse sentido, os altos índices de tuberculose são descritos em diversas pesquisas, em algumas, os valores se assemelhando com os valores encontrados nesta pesquisa, entretanto os valores de evolução para cura são satisfatórios.

\section{Conclusão}

A predominância dos casos foi constatada nos indivíduos do sexo masculino, com baixa escolaridade, da raça parda, na faixa etária de 20-39 anos, residentes na zona urbana, acometidos majoritariamente com a forma pulmonar da tuberculose, apresentando sorologia negativa para HIV e que evoluíram para cura posteriormente, sendo 2018 e 2019 os anos que registraram os maiores números de notificações de casos confirmados e a queda dos casos no ano de 2020, pode estar associada à pandemia, pois durante o ano, houve redução nos serviços de diagnósticos de tuberculose.

Diversos fatores podem se relacionar com os dados obtidos, mas foi constatada que a tuberculose anda é um problema de saúde pública, considerando a alta incidência nos números obtidos ao longo do período de estudo e fica em destaque a importância da utilização de indicadores epidemiológicos, que repercutem o quadro real das condições de saúde da população. Frente a isso, é relevante a atuação dos profissionais envolvidos diretamente nos programas de controle e combate da tuberculose, e a viabilização do desenvolvimento estrutural e estratégico, a fim de melhorar os serviços oferecidos e garantir a saúde pública e controle de doenças.

A descrição de um perfil epidemiológico possibilita a identificação do comportamento da tuberculose no contexto do estudo, e pode contribuir com o desenvolvimento das estratégias aplicadas no controle e combate da tuberculose. Portanto, diante dos resultados obtidos, ressalta-se o incentivo de melhorias nos planejamentos através dos profissionais envolvidos que lidam com o desenvolvimento e orientações, para que resulte na melhoria da saúde pública.

Nota-se a presença de casos relatados como ignorados ou em branco nos registros analisados na pesquisa, pois a realização dos estudos epidemiológicos depende da qualidade dos dados registrados pelos profissionais responsáveis, que visam obter precisão nos dados e possibilitar um melhor acompanhamento e controle sobre os casos notificados.

Conclui-se que a conscientização sobre a tuberculose e o perfil obtido através do estudo, é fundamental para o aprimoramento da saúde pública, ressaltando a educação em saúde, que pode contribuir com a redução de novos casos e evolução para cura dos pacientes infectados, aumentando a qualidade de vida da população como um todo. 


\section{Referências}

Brasil. (2019). Ministério da Saúde. Secretaria de Vigilância em Saúde. Departamento de Vigilância das Doenças Transmissíveis. Manual de Recomendações para o Controle da Tuberculose no Brasil.

Rolla, V. (2013). Tuberculose. Agência Fiocruz de Notícias.

Frasão, G. (2020). Brasil reduz em $8 \%$ o número de mortes por tuberculose na última década. Departamento de Doenças de Condições Crônicas e Infecções Sexualmente Transmissíveis.

Barreira, D. (2018). Os desafios para a eliminação da tuberculose no Brasil. Epidemiologia e Serviços de Saúde, 27, e00100009.

Barreto, A. M. W., et al. (2014). Adoecimento. In: Procópio, M.J., org. Controle da tuberculose: uma proposta de integração ensino-serviço [online]. Editora FIOCRUZ, 120-144.

Barreto, M. T. Silva et al. (2020). Epidemiologia da tuberculose em um estado do nordeste brasileiro. Research, Society and Development, 9, e52973643e52973643.

Barreto, M. L., Pereira, S. M., \& Ferreira, A. A. (2016). BCG vaccine: efficacy and indications for vaccination and revaccination. Jornal de pediatria, 82, s45-s54.

Barros, P. G. de et al. (2017). Perfil Epidemiológico dos casos de Tuberculose Extrapulmonar em um município do estado da Paraíba, 2001-2010. 22, 343350 .

Brasil. 2020. Ministério da Saúde. Secretaria de Vigilância em Saúde. Departamento de Doenças de Condições Crônicas e Infecções Sexualmente Transmissíveis. Boletim Epidemiológico Especial.

World health organization (2017). Global tuberculosis report 2017.

Silva, É. A., \& Silva, G. A. da. (2016). O sentido de vivenciar a tuberculose: um estudo sobre representações sociais das pessoas em tratamento. Physis: Revista de Saúde Coletiva, 26, 1233-1247.

Nobre, C. V., Morais, H. C. C. (2019). tuberculose em algumas cidades do ceará: análise de dados de notificação no período de 2014-2018. Mostra Interdisciplinar do curso de Enfermagem.

Zagmignan, A., et al. (2014). Caracterização epidemiológica da tuberculose pulmonar no Estado do Maranhão, entre o período de 2008 a 2014 . Rev Investig Bioméd [internet], 6, 2-9.

Fontes, G. J. F., et al. (2019). Perfil epidemiológico da tuberculose no Brasil no período de 2012 a 2016. Revista Brasileira de Educação e Saúde, 9, 19-26.

Oliveira, M. S. R. et al. (2018). Perfil epidemiológico dos casos de tuberculose no estado do Maranhão nos anos de 2012 a 2016. Revista Prevenção de Infecção e Saúde, 4.

Dantas, H. M. et al. (2019). Análise epidemiológica dos casos notificados de tuberculose no brasil. o que as pesquisas revel e as pesquisas revelam sobre a saúde no brasil.

Alves, W. A. et al. (2017). Perfil epidemiológico dos casos notificados de tuberculose no Piauí nos anos de 2010 a 2014 . Revista Interdisciplinar, 10, 31-38.

Oliveira, G. do C. A. et al. (2021). Perfil epidemiológico da população com tuberculose no Estado do Rio de Janeiro. Rev. Pesqui. (Univ. Fed. Estado Rio J., Online), p. 197-204.

Vidal, L. (2021). Casos de tuberculose caem no Brasil, pandemia pode ter reduzido diagnóstico. Viva Bem.

Macedo, J. L. et al. (2018). Perfil epidemiológico da tuberculose em um Município do Maranhão. Revista Ciência \& Saberes-UniFacema, 3 , $699-705$.

Capone, D. et al. (2006). Tuberculose extrapulmonar. Revista Hospital Universitário Pedro Ernesto, 5(2).

Costa, R. R. da et al. (2018). Comparação entre os métodos de Ogawa-Kudoh e Petroff modificado para o cultivo de micobactérias no diagnóstico da tuberculose pulmonar. Einstein, 16.

Lira, J. L. M. et al. (2021). Perfil epidemiológico dos casos de tuberculose no Estado de Pernambuco de 2009 a 2019. Research, Society and Development, 10, e3710312916-e3710312916.

Ferreira, W. S., et al. (2021). Perfil clínico-epidemiológico dos casos de tuberculose com coinfecção HIV no município de Belém. Revista Eletrônica Acervo Saúde, 13, e5970-e5970.

Magno, E. da S., et al. (2017). Fatores associados à coinfecção tuberculose e HIV: o que apontam os dados de notificação do Estado do Amazonas, Brasil, 2001-2012. Cadernos de Saúde Pública, 33, e00019315.

De Miranda, L. O. et al. (2017). Aspectos epidemiológicos da coinfecção Tuberculose/HIV no Brasil: revisão integrativa. Revista Prevenção de Infecção e Saúde, 3.

Braga, F. G. (2019). Tuberculose: Análise epidemiológica em um município da Amazônia Legal. 